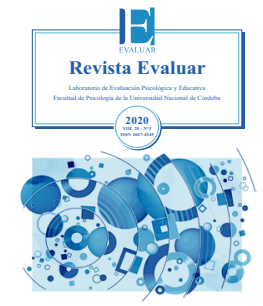

\title{
Test de Raven, baremos argentinos para el rango 19-30 años y efecto Flynn
}

\section{Raven's Progressive Matrices, Argentinean Norms for ages 19 to 30 and Flynn Effect}

\author{
Lilia Rossi-Casé ${ }^{1}$, Stella Maris Doná ${ }^{1}$, Ramiro Garzaniti ${ }^{1 *}$, Bruno Biganzoli ${ }^{1}$ \\ 1 - Facultad de Psicología de la Universidad Nacional de La Plata.
}

\author{
Introducción \\ Método \\ Resultados \\ Discusión \\ Referencias
}

Recibido: 19/12/2019 Revisado: 06/02/2020 Aceptado: 25/02/2020

\section{Resumen}

El objetivo de este artículo es presentar los resultados definitivos del proyecto de investigación dedicado a la construcción de baremos para el Test de Raven, Escala General (Raven, Raven, \& Court, 2003), para las edades de 19 a 30 años en la ciudad de La Plata, Argentina. La muestra estuvo conformada por 979 participantes y se dividió en cuatro intervalos de edad. Los resultados mostraron que a medida que aumenta la edad de los participantes, el rendimiento promedio de los grupos mejora; y que la heterogeneidad mostrada en sus respuestas resulta similar entre grupos. No se encontraron diferencias estadísticamente significativas entre los rendimientos de ambos sexos, ni entre los resultados actuales y los baremos del año 2000. Se constata una detención del incremento de puntajes esperable según el efecto Flynn. Se ensayan discusiones en torno a las diferencias cognitivas intergeneracionales que podrían originar este fenómeno, y se compara estos resultados con los obtenidos en otros países.

Palabras clave: Test de Raven, baremos, Argentina, 19-30 años, efecto Flynn

\begin{abstract}
This article aims to show the final results of the research project devoted to build norms for Raven's Standard Progressive Matrices (Raven, Raven, \& Court, 2003), on subjects aged 19 to 30 years old in the city of La Plata, Argentina. The sample consisted of 979 subjects of both sexes, and was divided into four age groups. The results show that as the participant's age increases, the average group's performance improves, and variability remains similar between groups. There were no statistically significant differences found between sexes, or between the current results and those from the 2000 norms. It was found that the scores' increase, expected according to the Flynn effect, has stopped, and the possible intergenerational cognitive differences which could explain this phenomenon are discussed. These results are also compared with those from recent research made in other countries.
\end{abstract}

Key words: Raven's Progressive Matrices, norms, Argentina, ages 19-30, Flynn effect

\footnotetext{
* Correspondencia a: Ramiro Garzaniti. E-mail: ramiro.garzaniti.unlp@gmail.com

Cómo citar este artículo: Rossi-Casé, L., Doná, S. M., Garzaniti, R., \& Biganzoli, B. (2020). Test de Raven, baremos argentinos para el rango 19-30 años y efecto Flynn. Revista Evaluar, 20(1), 17-31. Recuperado de https://revistas.unc.edu.ar/index.php/revaluar
} 


\section{Introducción}

El Test de Matrices Progresivas de Raven (en adelante Test de Raven) fue publicado por primera vez en el año 1938 por John C. Raven (Rossi-Casé, et al. 2016). Se trata de un test no verbal, de capacidad intelectual, de habilidad mental general. Examina el factor $g$, que surge de la Teoría Ecléctica de los dos Factores, propuesta por Charles Spearman en 1904 (Raven, Raven, \& Court, 2003). Con este fin, pone en juego procesos de educción de relaciones y correlaciones sobre un material en el que las variables no son obvias, de modo que se deben extraer nuevas comprensiones a partir de la información dada (Raven et al., 2003). Si bien hay investigaciones que afirman que los puntajes del test no solo reflejan el factor $g$, sino también otros factores como la capacidad visoespacial, la motivación y las estrategias de resolución de problemas (Gignac, 2015; Hayes, Petrov, \& Sederberg, 2015), Raven es considerado el test con mayor saturación de factor $g$ (Gignac, 2015).

Se trata de un test que mide la inteligencia fluida, por lo que coincidimos con Fernández-Liporace, Ongarato, Saavedra y Casullo (2004, p. 52) al afirmar que "esto explica la utilización tan difundida de las Matrices en los ámbitos de investigación", ya que los resultados que aporta permiten comparar poblaciones y/o sujetos que "han sido expuestos a situaciones educativas formales e informales de naturaleza bien disímil".

A partir del análisis de baremos de diversos tests de inteligencia y de los datos obtenidos de casi 50 mil participantes por internet, Hartshorne y Germine (2015) afirman que no hay una única edad en la que las personas logran un desempeño máximo en todas las tareas, o en la mayoría de ellas. Parten de la ya mencionada separación entre inteligencia fluida e inteligencia cristalizada, de las cuales suele entenderse que la primera alcanza su punto máximo en la adultez temprana, mientras que la segunda lo hace en la mediana edad.

Los autores refieren que hay una gran variabilidad en estas edades de desempeño máximo entre las diversas tareas que involucra cada uno de estos dos tipos de inteligencia (por ejemplo, memoria a corto plazo para nombres o rostros en el caso de la inteligencia fluida, vocabulario e información en el de la cristalizada), y por ese motivo la distinción entre inteligencia fluida $\mathrm{y}$ cristalizada en cuanto a un momento de desempeño máximo ya no sería relevante. No obstante, el estudio del cambio de los puntajes en los tests de inteligencia a través de las generaciones ha mantenido vivo el interés en estos estudios en la actualidad.

El uso generalizado de los tests de inteligencia demostró que los puntajes medios obtenidos aumentaban regularmente y de manera notable con el correr del tiempo en todo el mundo (Flynn, 1984; Rossi-Casé, Neer, \& Lopetegui, 2001). Esta constatación indica que, para la misma población, las normas para el cociente intelectual se vuelven obsoletas conforme pasan los años. Es decir que en la actualidad, para obtener el mismo puntaje transformado que hace algunas décadas, las puntuaciones directas deberían ser más altas, debiendo resolverse un mayor número de problemas. Estos aumentos son mayores en aquellos tests que miden la inteligencia fluida (Sundet, Barlaug, \& Torjussen, 2004). Esto llevó a pensar que el aumento está relacionado al factor $g$ de la inteligencia y no a los factores específicos (i.e., factores $e$ ).

En su estudio inicial, Flynn (1984) estableció que la magnitud de aumento del cociente intelectual, medido inicialmente con las escalas Wechsler y Stanford-Binet, era de 0.3 puntos por año o 3 puntos por década. Estos estudios se realizaron comparando muestras estadounidenses 
entre los años 1932 y 1978 . Más adelante, la misma tendencia se corroboró con datos de otros 20 países (Flynn \& Rossi-Casé, 2012; Gignac, 2015; Sundet et al., 2004; Teasdale \& Owen, 2007).

Con respecto a la Argentina, este equipo realizó una primera constatación del efecto Flynn en la ciudad de La Plata y alrededores al actualizar los baremos del Test de Raven, Escala General, en el año 2000, y compararlos con la estandarización del año 1964 (Rossi-Casé et al., 2001). Esta comparación mostró un considerable aumento en los puntajes directos del test para todas las edades (Flynn \& Rossi-Casé, 2011, 2012; Rossi-Casé, Neer, \& Lopetegui, 2002, 2017; Rossi-Casé et al., 2014, 2016). No obstante, durante la década siguiente, empezó a constatarse que dichos puntajes aumentaban a un ritmo mucho menor.

La mencionada desaceleración en el aumento de los puntajes fue observada por vez primera en los países escandinavos. Tal es el caso de investigaciones longitudinales hechas en Noruega (Sundet et al., 2004), en las cuales se muestra una disminución en los puntajes medios de una batería de tests administrados a los jóvenes de 18 años, antes de entrar al servicio militar, desde los años 50. Las pruebas utilizadas son tests de matemática y lenguaje, similares a los subtests del WAIS IV (Wechsler, 2008), y un test no verbal que fue construido de forma similar al Test de Raven. Los dos primeros miden la inteligencia cristalizada, mientras que el último mide la inteligencia fluida.

Teasdale y Owen (2007) estudiaron los datos aportados por una batería similar en Dinamarca, la cual desde 1957 se administra a todos los jóvenes de 18 años que ingresan al servicio militar. De los cuatro tests que la componen, los autores afirman que el de Matrices de Letras es el más similar a las Matrices Progresivas de Raven. Aquí encontraron que, si bien hubo un pequeño incremento de los puntajes entre 1988 y 1998, los mismos disminuyeron en 2003-2004, incluso por debajo de los obtenidos en 1988. Esto se observó en jóvenes de todos los niveles educativos. Con estos datos, los autores apoyan la afirmación de que en lo que va del siglo XXI ha habido muy poca evidencia sobre la continuidad del efecto Flynn, tal como se lo define hasta este momento, es decir, como el aumento continuo de las medias en los tests de inteligencia. Este mismo efecto meseta fue constatado en los puntajes del Test de Raven en la ciudad de La Plata, Argentina, para las edades 13-18 años por este equipo de investigación (Rossi-Casé et al., 2016).

Cuando se realizaron las primeras observaciones del efecto Flynn surgieron diversas hipótesis explicativas. De todas formas, la causa de este fenómeno permanece aún desconocida y no existe evidencia suficiente que permita afirmar que este hecho refleje un verdadero aumento de la inteligencia. Entre las hipótesis propuestas se encuentran las siguientes: la heterosis, es decir, la mejora en las condiciones de vida en las distintas poblaciones y una alimentación o nutrición más adecuada; la expansión del sistema educativo; la reducción del tamaño de las familias nucleares, con el consecuente incremento del desarrollo psicológico; la adquisición progresiva de ciertas habilidades para responder satisfactoriamente a los tests; un mayor desarrollo de la escolarización y educación de los niños, y la creciente tecnologización de la cultura, desde los juegos de video hasta el acceso cada vez más irrestricto a los medios de comunicación a través de internet, que ofrecen otros aspectos de estimulación (Sundet et al., 2004; Baker et al., 2015; Passig, 2015). Investigaciones realizadas en países que han sufrido guerras podrían ser consideradas casos que sostienen estas hipótesis. Tal es el caso de Kuwait, Sudán y Siria (Dutton, Bakhiet, Alhossein, \& Essa, 2019; Dutton, Bakhiet et al., 2018; Dutton, Essa et al., 2018), donde luego de conflictos militares (la Primera Guerra del Golfo en el caso de 
Kuwait y la Guerra Civil en Sudán y Siria) que trajeron como consecuencia un detrimento en la calidad de vida de sus habitantes, se detectó una baja en los puntajes de los test de inteligencia. Estos descubrimientos llevaron a parte del equipo de investigación a suponer una correlación positiva entre el efecto Flynn y el crecimiento del PBI de un país. Dicha suposición fue corroborada por Rindermann y Becker (2018).

Por su parte, Armstrong y Woodley (2014) afirman que hay evidencia neurológica que señala que el efecto Flynn está asociado a un aumento en el tamaño del cerebro que ha potenciado las funciones del hipocampo, aunque no descartan que otra causa para este fenómeno sea la posibilidad de que los tests que miden la inteligencia fluida y la capacidad eductiva, como el Test de Matrices Progresivas de Raven, contengan algún tipo de andamio cognitivo que oriente el uso de habilidades no dependientes del factor $g$ para resolver los problemas. Es decir, que el mismo test podría guiar al sujeto en la adquisición de dichas habilidades.

La observación de una detención en el aumento de los puntajes medios en los tests nos invita a una nueva consideración de las posibles causas de este fenómeno. En un intento por abordar esta cuestión, Bratsberg y Rogeberg (2018), a partir de datos similares a los empleados por Sundet et al. (2004), pusieron a prueba las hipótesis causales para el efecto Flynn y su reverso, clasificándolas en dos grupos: aquellas que apelan al origen genético de este efecto y las que postulan una causalidad ambiental. Al no encontrar diferencias significativas en el desarrollo de este efecto entre sujetos pertenecientes a la misma familia con respecto a la población general, los autores concluyen que el primer grupo de hipótesis carece de sustento suficiente, y se decantan entonces por el segundo. A través de un relevamiento realizado con 70 expertos en la materia, Rindermann,
Becker y Coyle (2017) arribaron a la misma conclusión.

En este punto, consideramos que las perspectivas ligadas a las dimensiones generacionales pueden aportar elementos para elucidar la presentación actual del efecto Flynn. Los sujetos que integran la muestra del presente estudio se corresponden con las denominadas Generación Y y $\mathrm{Z}$, la cohorte de personas nacidas en la década de los años 80 y de los años 90, respectivamente (Strauss \& Howe, 1991). Navós (2014) retoma la tradicional clasificación generacional según el criterio de año de nacimiento y establece los siguientes grupos: a) Tradicionalistas: nacidos antes de 1945; b) Baby boomers: nacidos entre 1945 y 1965; c) Generación X: nacidos entre 1966 y 1980; d) Generación Y: nacidos entre 1981 y 1995, los famosos millennials; e) Generación Z: nacidos a partir de 1996.

Ahora bien, dichos grupos suponen, según nos plantea el autor, diferencias relativas al contexto social y político en el cual nacieron y se criaron (códigos, aspiraciones, valores), diferencias que actualmente coexisten en diversos ámbitos de nuestra sociedad. En efecto, Navós (2014) resume las características correspondientes a los grupos generacionales de la siguiente manera:

Los tradicionalistas valorizan fundamentalmente la educación y el esfuerzo en tanto camino hacia el progreso; la palabra y la autoridad denotan para ellos respeto tanto en las relaciones familiares como también frente a las instituciones, jerarquías y superiores, y procuran la proyección a largo plazo durante sus vidas, etc.

El grupo generacional baby boomers se constituyó en un contexto de mayor incertidumbre tras el paso de las dos Guerras Mundiales. En ese sentido, bajo los efectos de las coyunturas políticas y sociales, comienzan a discutir las jerarquías, lo establecido, buscan lograr un título y profesionalizarse a los fines de alcanzar un me- 
nor grado de dependencia, se oponen a los valores impuestos y apuestan a la paz y la libertad.

La generación $X$ se compone de sujetos competitivos e independientes, que aceptan la diversidad y procuran contextos informales. Buscan su autodesarrollo, tomando dimensión de la inmediatez de sus vidas $y$, en consecuencia, acelerando sus ritmos profesionales, procurando el disfrute de otros planos sociales.

El grupo generacional Y o millennials atravesó un contexto signado por la globalización y el acceso a internet. Las tecnologías se presentan para ellos como inherentes a sus crianzas y prácticas cotidianas. Están siempre conectados y tienen la posibilidad de acceder a información y a realidades más amplias que las generaciones anteriores. Priorizan lo inmediato por sobre la construcción de proyectos a largo plazo. Procuran disfrutar de una buena calidad de vida, de lo que hacen, buscar nuevos horizontes, sostener un pensamiento independiente por fuera de los patrones establecidos.

Por último, la Generación $Z$ se caracteriza por haber nacido en una época en donde el consumo adquiere su mayor auge y el acceso a las tecnologías se presenta desde el nacimiento. $\mathrm{Su}$ comunicación se encuentra predominantemente mediada por herramientas digitales. La inmediatez signa sus vidas, y priorizan el disfrute por sobre el trabajo.

De esta manera, los procesos histórico-sociales y los desarrollos tecnológicos se sintetizan en patrones de comportamiento específicos en cada grupo generacional. Con el arribo y difusión masiva de la tecnología digital a fines del siglo $\mathrm{XX}$, se inaugura una discontinuidad generacional substancial, identificada por Prensky (2001) con la oposición entre nativos e inmigrantes digitales. Prensky designa nativos digitales a quienes han nacido y se han formado utilizando la particular "lengua digital" de juegos por ordenador, vídeo e Internet (tales como las generaciones $\mathrm{Y}$ y Z) en tanto que a quienes no han nacido en ese contexto, pero que deben ajustarse en la actualidad a su uso, los denomina inmigrantes digitales. En ese sentido, más allá de las diferencias entre las generaciones, encontramos entre ambos grupos un modo de pensar y procesar la información significativamente diferente. Los nativos digitales se caracterizan por un acceso rápido e inmediato a la información dadas las disponibilidades que genera internet, optando por textos que disminuyan la complejidad del conocimiento y tiendan a ser intuitivos.

Sartori (1998) señala que la televisión y el conjunto de nuevas tecnologías, que se inscribieron en la crianza de quienes hoy se presentan como generación $\mathrm{Y}$, altera, empobrece el aparato cognoscitivo del homo sapiens y menoscaba la naturaleza simbólica del hombre. Dado que la televisión ocupó un lugar privilegiado en la crianza de quienes componen la generación millennial, se presenta una prevalencia del ver sobre el hablar, con lo que arribamos a una sustitución que modifica profundamente la relación entre entender $\mathrm{y}$ ver ya que actualmente el relato (su explicación) está supeditado a las imágenes que aparecen en la pantalla. Esto, según señala Sartori, reduce la capacidad de abstracción y entendimiento. En ese sentido, el autor postula un proceso de suplantación del homo sapiens por el homo videns, en tanto en este último predomina el lenguaje perceptivo por sobre otras capacidades cognoscitivas.

Las características enumeradas por estos autores convergen en referencia a la inmediatez como elemento que signa la vida de estos sujetos. Esto remite a los desarrollos de Bauman (2002) sobre la modernidad líquida. Este autor se apoyó en la cualidad que los líquidos tienen para cambiar su forma y para desplazarse, por oposición a los sólidos, como metáfora para referirse al impacto que ha tenido en la sociedad y en el individuo la 
pérdida actual de ciertos códigos y conductas que solían servir como puntos estables de orientación. Este autor asocia la cualidad de "líquido" con las ideas de levedad, movilidad e inconstancia. La forma que un líquido tenga dependerá del momento en que se lo observe, por lo que "para ellos lo que cuenta es el flujo del tiempo más que el espacio que pueden ocupar" (Bauman, 2002, p. 8). Más adelante en su obra habla del síndrome de la impaciencia, producto de la concepción del acceso a los atajos como emblema de privilegio en la escala social. Según el autor, la posición de cada uno en dicha escala "se mide por la capacidad (o la ineptitud) para reducir o hacer desaparecer por completo el espacio de tiempo que separa el deseo de su satisfacción" (Bauman, 2008, p. 22).

El objetivo de este artículo es presentar los resultados finales del proyecto de investigación dedicado a la construcción de normas de percentiles para el Test de Matrices Progresivas de Raven, Escala General, para las edades de 19 a 30 años en la ciudad de La Plata, Argentina. De modo complementario, y sobre la base de reflexiones introducidas anteriormente (Rossi-Casé, Doná, Garzaniti, Biganzoli, \& Llanos-Barja, 2018; Rossi-Casé, Doná, Biganzoli, \& Garzaniti, 2019), se elaborarán algunas consideraciones sobre las particularidades de la inteligencia en las nuevas generaciones. Las mismas se desprenden de la indagación de las diferencias entre las poblaciones en las cuales el efecto Flynn estaba aún vigente y las actuales, en las que el mismo ya no se observa.

\section{Método}

Participantes

La muestra estuvo conformada por 979 participantes de ambos sexos, 543 mujeres y 426 varones, con edades comprendidas dentro del rango de 19 a 30 años, considerando la edad en años cumplidos al momento de la administración del test. El total de casos quedó determinado una vez que fueron desestimados los protocolos con discrepancias significativas de los puntajes directos.

Según la edad, se dividió la muestra en cuatro intervalos: 19-20 años, 21-22 años, 23-24 años y 25-30 años. La amplitud de los rangos de edad obedece al hecho constatado de que los puntajes de la prueba se incrementan con el aumento de edad, en intervalos iguales de dos años desde los 13 a los 24 años, y en intervalos de edad diferentes, a partir de los 25 años. La versión original de la prueba y las revisiones argentinas del año 1964 y 2000 presentan esta segmentación de edades, por lo que mantenerlas nos permite realizar comparaciones de los resultados alcanzados (Raven et al., 2003; Rossi-Casé et al., 2001, 2017).

Además de ser caracterizada por la edad, la muestra fue estratificada con respecto a la variable sexo, teniendo como referencia los datos del último censo poblacional para la ciudad de La Plata y sus alrededores de la República Argentina, realizado en el año 2010 (Instituto Nacional de Estadística y Censos [INDEC], 2012). Todas las personas que conformaron la muestra, habían alcanzado el nivel de escolarización media.

\section{Instrumentos}

Se utilizó el Test de Matrices Progresivas de Raven; Escala General (Raven, 2005). Es una prueba no verbal, independiente del lenguaje hablado o escrito, de la cultura o escolarización de las personas. Esta prueba mide la capacidad intelectual general mediante la comparación de formas y el razonamiento por analogía, sin intervención de los conocimientos adquiridos previamente. Brinda información sobre la capacidad intelectual, midiendo dos de sus componentes: educción, en tanto razonamiento lógico; y repro- 
ducción de la información.

El test está compuesto por sesenta problemas organizados en cinco series (A; B; C; D; E) de doce ítems cada una. Cada una de las series comienza con problemas sencillos y luego paulatinamente va aumentando la complejidad. A su vez, la complejidad de las series también va en aumento, desde la serie A hasta la E. La puntuación directa máxima es de 60 puntos.

Tabla 1

Composición de la muestra.

\begin{tabular}{|c|c|c|c|}
\hline \multirow[t]{2}{*}{ Edad } & \multicolumn{2}{|c|}{ Sexo } & \multirow{2}{*}{$\begin{array}{c}\text { Total de } \\
\text { casos por } \\
\text { edad }\end{array}$} \\
\hline & Mujer & Varón & \\
\hline 19-20 años & 203 & 133 & 336 \\
\hline 21-22 años & 116 & 107 & 223 \\
\hline 23-24 años & 121 & 113 & 234 \\
\hline 25-30 años & 103 & 83 & 186 \\
\hline $\begin{array}{c}\text { Total de casos } \\
\text { por Sexo }\end{array}$ & 543 & 436 & 979 \\
\hline
\end{tabular}

Nota. $N=979$.

\section{Procedimiento}

Se obtuvo la autorización previa de los participantes en la investigación, mediante un formulario de consentimiento informado, el cual incluía una explicación del propósito de la investigación, una aclaración sobre la confidencialidad de los datos, y un agradecimiento por la participación.

El test se administró de manera individual o grupal, según el caso, con presencia del examinador y sin límite de tiempo para su ejecución. Sin embargo, se observó que los participantes más rápidos completaron la prueba dentro del rango de 30 minutos, y los participantes más lentos, dentro del rango de 60 minutos.
Para la consigna, se siguieron de manera rigurosa las instrucciones dadas por el autor para la autoadministración o administración colectiva. La prueba se tomó en distintos períodos de los años 2016 a 2019: junio a noviembre de 2016, marzo a septiembre de 2017, marzo a agosto de 2018, y abril a agosto de 2019. La administración estuvo a cargo de este Equipo de Investigación. El número mayor de participantes por grupo fue de 32. La mayoría de las pruebas fueron realizadas en el espacio físico de la Facultad de Psicología de la Universidad Nacional de La Plata, lugar al que los participantes fueron citados por ser la institución a la que se vincula esta investigación.

Al conformar la base de datos, se excluyeron 58 protocolos que mostraban una discrepancia mayor a la esperada en la composición de los puntajes. Se analizó una base de datos que quedó conformada por 979 protocolos.

\section{Procesamiento de los datos}

Con la información recogida se construyó una base utilizando la planilla de cálculos Microsoft Excel (Microsoft Corporation, 2010). Luego se exportaron los datos al software InfoStat (Di Rienzo et al., 2018) para obtener los estadísticos descriptivos que permitieron elaborar las normas para cada grupo de edad.

Se definió un intervalo de confianza del 95\%, y se estableció que el error muestral máximo sería del 3\% para el tamaño de esta muestra. Los resultados obtenidos en el estudio actual se compararon con los baremos obtenidos en los años 1964 y 2000, para los mismos grupos etarios de la ciudad de La Plata, Argentina, a excepción de las puntuaciones directas obtenidas en el año 1964 para el rango de edad 25-30 años, que no se encuentran disponibles (Rossi-Casé et al., 2017).

La comparación de dichos rendimientos 
promedio por rango de edad y por sexo se realizó aplicando el estadístico $z$ para la prueba de hipótesis de la diferencia de medias entre muestras aleatorias, normales e independientes, con un intervalo de confianza del 95\%.

\section{Resultados}

El análisis de las puntuaciones directas obtenidas muestra que en los cuatro grupos de edad se alcanzó la puntuación máxima posible (ver Tabla 2). La puntuación mínima observada fue de 34 puntos y se registró en el grupo de menor edad. Los resultados mostraron que, a medida que aumenta la edad de los participantes, el rendimiento promedio de los grupos mejora; y que la heterogeneidad de las respuestas resulta similar entre grupos.

La prueba $z$ de diferencia de medias entre dos muestras permitió observar que entre las puntuaciones promedio obtenidas en el año $2000 \mathrm{y}$ las del año 2019 no se constatan diferencias significativas. Para ningún rango de edad estudiado el estadístico $z$ calculado pudo superar el valor crítico de $z$, para un nivel de significación $\alpha=.05$. Las variaciones encontradas se deberían al azar.

De igual manera, los varones obtuvieron puntuaciones levemente superiores que las mujeres en todos los rangos de edad; con un mínimo de respuestas correctas también mayor, y alcanzando siempre la mayor puntuación posible. Las variaciones encontradas entre los rendimientos de ambos sexos no fueron estadísticamente significativas $(p<.05)$. Las diferencias encontradas también se deberían al azar.

Resultados para el grupo de edad de 19 a 20 años

En los participantes que integran este grupo
Tabla 2

Descripción de los promedios y variaciones de las puntuaciones directas, por rango de edades.

\begin{tabular}{cccc}
\hline $\begin{array}{c}\text { Rango } \\
\text { de Edades }\end{array}$ & Promedio & $\begin{array}{c}\text { Desviación } \\
\text { estándar }\end{array}$ & $\begin{array}{c}\text { Rango de } \\
\text { Respuestas }\end{array}$ \\
\hline 19-20 años & 50.16 & 4.77 & 34 a 60 ptos. \\
21-22 años & 51.78 & 4.39 & 40 a 60 ptos. \\
$23-24$ años & 51.51 & 5.52 & 35 a 60 ptos. \\
& & & \\
$25-30$ años & 52.89 & 4.83 & 38 a 60 ptos. \\
\hline
\end{tabular}

Nota. Puntuación máxima: 60 puntos . $\mathrm{N}=979$.

se observan los resultados más bajos de todos los que integran la muestra. La puntuación promedio es de 50.16 puntos y la desviación estándar es de 4.77 puntos. El rango de respuestas correctas fue de 34 para los puntajes más bajos y de 60 para los más altos. Entre las mujeres, el rango de respuestas correctas fue de 34 a 59, y entre los varones, de 37 a 60 .

El rendimiento de los varones $(\mathrm{M}=51.13$ puntos; $\mathrm{DE}=4.73$ puntos) es mejor que el de las mujeres $(\mathrm{M}=49.63$ puntos; $\mathrm{DE}=4.71$ puntos $)$. La prueba de diferencia de medias mostró que tales variaciones no son estadísticamente significativas $(p<.05)$.

La mitad de los sujetos que integran este grupo de edad ha obtenido 50 puntos o menos (ver Tabla 3). Si bien esto representa un incremento de 11 puntos respecto del baremo del año 1964 (Rossi-Casé et al., 2001), el resultado observado es 1 punto menor que la norma alcanzada en el año 2000 (Rossi-Casé et al., 2017). Este comportamiento muestra un incremento significativo de 
las puntuaciones respecto de las normas 1964 ( $p$ $<.05)$. En comparación con los baremos del año 2000 , se constata una igualdad para los percentiles 75 o superior, y una disminución en todos los valores percentilares de la mediana e inferiores.

\section{Tabla 3}

Edades 19-20 años: Comparación de la distribución percentilar de las puntuaciones directas, años 1964, 2000 y 2019.

\begin{tabular}{cccc}
\hline & \multicolumn{2}{c}{ Baremos } & Valores \\
Percentil & año 1964 & año 2000 & año 2019 \\
\hline P99 & 53 & 59 & 59 \\
P95 & 53 & 57 & 57 \\
P90 & 51 & 56 & 56 \\
P75 & 45 & 54 & 54 \\
P50 & 40 & 52 & 51 \\
P25 & 34 & 48 & 47 \\
P10 & 27 & 46 & 44 \\
P5 & 22 & 43 & 41 \\
\hline
\end{tabular}

Nota. Puntuación máxima: 60 puntos. $\mathrm{n}=336$.

Resultados para el grupo de edad de 21-22 años

La puntuación promedio observada es de 51.78 puntos y la desviación estándar es de 4.35 puntos (ver Tabla 4). El rango de respuestas correctas fue de 40 para los puntajes más bajos y de 60 para los más altos. Entre las mujeres, el rango de respuestas correctas fue de 40 a 59; y entre los varones, de 41 a 60.

El rendimiento de los varones $(\mathrm{M}=51.93$ puntos; $\mathrm{DE}=3.99$ puntos) es levemente mejor que el de las mujeres $(\mathrm{M}=51.67$ puntos; $\mathrm{DE}=$ 4.71 puntos), pero no se observa una diferencia de medias estadísticamente significativa $(p<.05)$.

La mitad de los participantes que integran este grupo de edad ha obtenido al menos 52 pun- tos. Esto representa un incremento de 12 puntos respecto del baremo del año 1964 (Rossi-Casé et al., 2001), y 1 punto respecto de la norma alcanzada en el año 2000 (Rossi-Casé et al., 2017). Este incremento significativo en las puntuaciones respecto de las normas 1964 se observó en todos los valores percentilares calculados $(p<.05)$. La comparación de resultados con los baremos del año 2000 demuestra que se conservan iguales los puntajes directos necesarios para alcanzar el percentil 90 y superiores; y aumentan en un punto los necesarios para alcanzar los percentiles $75 \mathrm{e}$ inferiores.

\section{Tabla 4}

Edades 21-22 años: Comparación de la distribución percentilar de las puntuaciones directas, años 1964, 2000 y 2019.

\begin{tabular}{cccc}
\hline & \multicolumn{2}{c}{ Baremos } & $\begin{array}{c}\text { Valores } \\
\text { percentilares } \\
\text { Percentil }\end{array}$ \\
& año 1964 & año 2000 & año 2019 \\
\hline P99 & 53 & 59 & 59 \\
P95 & 53 & 58 & 58 \\
P90 & 51 & 57 & 57 \\
P75 & 45 & 54 & 55 \\
P50 & 40 & 51 & 52 \\
P25 & 34 & 48 & 49 \\
P10 & 27 & 45 & 46 \\
P5 & 22 & 42 & 43 \\
\hline
\end{tabular}

Nota. Puntuación máxima: 60 puntos. $\mathrm{n}=223$.

Resultados para el grupo de edad de 23 a 24 años

En los participantes que integran este grupo se observan los resultados con mayor variabilidad de todos los que integran la muestra. La puntuación promedio es de 51.51 puntos y la desviación estándar de 5.52 puntos (ver Tabla 5). El rango de respuestas correctas fue de entre 35 y 59 en mujeres; y entre 37 y 60 entre varones. 
El rendimiento de los varones $(\mathrm{M}=53.76$ puntos; $\mathrm{DE}=5.55$ puntos) es mejor que el de las mujeres $(\mathrm{M}=50.18$ puntos; $\mathrm{DE}=5.09$ puntos $)$. La diferencia de medias muestra que los rendimientos promedio no son estadísticamente significativos y que tales variaciones se deberían al $\operatorname{azar}(p<.05)$.

La mitad de los sujetos que integran el grupo de edad ha obtenido 53 puntos o menos. El resultado observado representa un incremento de 13 puntos respecto del baremo del año 1964 (Rossi-Casé et al., 2001), al tiempo que permanece igual respecto de la norma alcanzada en el año 2000 (Rossi-Casé et al., 2017). Este incremento significativo de las puntuaciones respecto de las normas $1964(p<.05)$ se observó en todos los valores percentilares calculados. Respecto de los baremos del año 2000, no hay aumento de las puntuaciones directas sino que se constata la igualdad o, incluso, la disminución de 1 y 2 puntos para los valores de los percentiles 99 y 25 , respectivamente.

Resultados para el grupo de edad de 25 a 30 años

En los sujetos que integran este grupo se observan los resultados más altos de todos los que integran la muestra. La puntuación promedio es de 52.89 puntos, y la desviación estándar de 4.83 puntos (ver Tabla 6). El rango de respuestas correctas fue de 38 para mujeres y 42 en varones; hasta los 60 puntos para los más altos, valor alcanzado en ambos sexos.

La mitad de los sujetos que integran el grupo de edad ha obtenido 54 puntos o menos. El resultado observado representa un incremento de 4 puntos respecto del baremo del año 2000 (Rossi-Casé et al., 2017).

El rendimiento de los varones $(\mathrm{M}=55.07$ puntos; $\mathrm{DE}=4.84$ puntos) es mejor que el de las

\section{Tabla 5}

Edades 23-24 años: Comparación de la distribución percentilar de las puntuaciones directas, años 1964, 2000 y 2019.

\begin{tabular}{cccc}
\hline & \multicolumn{2}{c}{ Valores } \\
Percentil & \multicolumn{2}{c}{ Baremos } & percentilares \\
& & & \\
& año 1964 & año 2000 & año 2019 \\
\hline P99 & 53 & 60 & 59 \\
P95 & 53 & 59 & 59 \\
P90 & 51 & 58 & 58 \\
P75 & 45 & 55 & 56 \\
P50 & 40 & 53 & 53 \\
P25 & 34 & 49 & 47 \\
P10 & 27 & 44 & 44 \\
P5 & 22 & 42 & 42 \\
\hline
\end{tabular}

Nota. Puntuación máxima: 60 puntos. $n=234$.

mujeres $(\mathrm{M}=51.76$ puntos; $\mathrm{DE}=4.44$ puntos $)$, aunque la diferencia de medias no es estadísticamente significativa $(p<.05)$.

Respecto de dichos baremos, se constata el aumento de al menos 1 punto en todos los percentiles más altos; y de 4 ó 5 puntos para los valores percentilares medio y más bajos.

\section{Baremos}

La Tabla 7 presenta los baremos 2019 del Test de Raven, Escala General, para las edades de 19 a 30 años, para la ciudad de La Plata, Argentina.

\section{Discusión}

En las casi dos décadas transcurridas desde la elaboración de las normas anteriores del test hasta la actualidad, los resultados obtenidos 
Tabla 6

Edades 25-30 años: Comparación de la distribución percentilar de las puntuaciones directas, años 2000 y 2019.

\begin{tabular}{ccc}
\hline Percentil & $\begin{array}{c}\text { Baremos } \\
\text { año 2000 }\end{array}$ & $\begin{array}{c}\text { Valores percentilares } \\
\text { año 2019 }\end{array}$ \\
\hline P99 & 59 & 60 \\
P95 & 58 & 59 \\
P90 & 57 & 58 \\
P75 & 55 & 57 \\
P50 & 50 & 54 \\
P25 & 45 & 50 \\
P10 & 42 & 47 \\
P5 & 39 & 43 \\
\hline
\end{tabular}

Nota. Puntuación máxima: 60 puntos. $\mathrm{n}=186$.

permiten mostrar, en consonancia con investigaciones realizadas en otros países (Bratsberg \& Rogeberg, 2018; Brouwers, van de Vijver, \& van Hemert, 2009; Flynn, 2013; Sundet et al., 2004; Teasdale \& Owen, 2007), una detención en el aumento de los puntajes directos necesarios para alcanzar el rendimiento promedio en cada rango de edad $(p<.05)$. Las variaciones encontradas entre los valores actuales y los baremos del año 2000 (Rossi-Casé et al., 2017) no resultaron ser estadísticamente significativas, por lo que se constata la detención del efecto Flynn, característico de la segunda mitad del siglo XX (ver Tabla 8).

Los autores antes citados han mencionado como posible causa de este fenómeno un efecto techo. Esto se podría explicar a partir de los mismos factores que se tuvieron en cuenta para intentar explicar el anterior aumento: heterosis, nutrición más adecuada, expansión del sistema educativo. Para fundamentarlo empíricamente, Brouwers et al. (2009), argumentaron que en los países con menor PBI per cápita los puntajes promedio seguían aumentando, mientras que el efecto techo podía observarse en los países escandinavos. De la misma forma, Teasdale y Owen (2007) afirmaron que los países en vías de desarrollo estarían lejos de llegar al efecto techo.

Sin embargo, la situación de Argentina representa un ejemplo del estancamiento de los puntajes promedio en un país no tan desarrollado y cuyos indicadores sociales están lejos de aquellos de los países escandinavos. Consideramos pertinente, en este punto, aclarar que efecto te-

\section{Tabla 7}

Edades 19-30 años: Escala percentilar y puntuaciones directas, año 2019, ambos sexos, para cada grupo etario.

\begin{tabular}{|c|c|c|c|c|}
\hline \multirow{2}{*}{ Percentil } & \multicolumn{4}{|c|}{ Edad } \\
\hline & 19-20 años & 21-22 años & 23-24 años & 25-30 años \\
\hline P99 & 59 & 59 & 59 & 60 \\
\hline P95 & 57 & 58 & 59 & 59 \\
\hline P90 & 56 & 57 & 58 & 58 \\
\hline P75 & 54 & 55 & 56 & 57 \\
\hline P50 & 51 & 52 & 53 & 54 \\
\hline P25 & 47 & 49 & 47 & 50 \\
\hline P10 & 44 & 46 & 44 & 47 \\
\hline P5 & 41 & 43 & 42 & 43 \\
\hline
\end{tabular}

Nota. Puntuación máxima: 60 puntos. $\mathrm{N}=979$. 
cho y efecto meseta no son sinónimos. El primero aparece cuando se han alcanzado los puntajes más altos que era posible obtener en la prueba, por lo que los mismos ya no pueden continuar aumentando. En cambio, el segundo se da cuando se produce un estancamiento en los puntajes sin haber alcanzado el máximo posible. Hecha esta diferenciación, observamos que mientras que lo descrito en los países escandinavos fue caracterizado como efecto techo, el caso argentino muestra más bien un efecto meseta.

El hecho de que la recolección de datos del presente estudio se haya realizado a lo largo de tres años podría acarrear el riesgo de la introducción de un sesgo indeseado en el análisis de los resultados, si se sostuviera la vigencia local del efecto Flynn. No obstante, el hecho de que aún en estos años los resultados no hayan mostrado diferencias significativas con respecto a aquellos de la estandarización anterior del Test, refuerza la constatación del efecto meseta, lejos de invalidarla.

Por otro lado, si bien el PBI per cápita es menor en Argentina que en los países donde se observó el efecto techo, la globalización ha logrado que las nuevas generaciones en el mundo oc- cidental accedan a los mismos medios de comunicación y redes sociales. Proponemos entonces retomar el concepto de homo videns, postulado por Sartori (1998), especialmente en lo atinente al empobrecimiento del aparato cognitivo, y considerar que, además, para estas nuevas generaciones la inmediatez es una virtud. La administración del Test de Raven puede tomar unos 40 minutos o más (requiriendo bastante esfuerzo cognitivo en las últimas series), lo cual podría generar un desgano a la hora de concluir con el test que explique el amesetamiento de los puntajes en el mismo.

Consideramos que una línea a indagar es si se encuentra una explicación posible del amesetamiento del efecto Flynn a partir de postulados de Piscitelli (2006), Sartori (1998) y Prensky (2001), entre otros, sobre los grupos generacionales y la discontinuidad substancial para los grupos $\mathrm{Y}$ y Z, ya que suponen cambios cognitivos signados por la inmediatez, como la disminución de las capacidades de abstracción y entendimiento, y nuevos modos de pensar y procesar la información que motivarían el desgano de estas generaciones frente al Test de Raven en tanto el mismo les demanda un esfuerzo cognitivo superior al que emplean en la actualidad, lo cual se manifestaría en los resul-

Tabla 8

Edades 19-30 años: Comparación de la distribución percentilar de las puntuaciones directas, años 2000 y 2019.

\begin{tabular}{|c|c|c|c|c|c|c|c|c|}
\hline \multirow{3}{*}{ Percentil } & \multicolumn{8}{|c|}{ Edad } \\
\hline & \multicolumn{2}{|c|}{ 19-20 años } & \multicolumn{2}{|c|}{ 21-22 años } & \multicolumn{2}{|c|}{ 23-24 años } & \multicolumn{2}{|c|}{ 25-30 años } \\
\hline & año 2000 & año 2019 & año 2000 & año 2019 & año 2000 & año 2019 & año 2000 & año 2019 \\
\hline P99 & 59 & 59 & 59 & 59 & 60 & 59 & 59 & 60 \\
\hline P95 & 57 & 57 & 58 & 58 & 59 & 59 & 58 & 59 \\
\hline P90 & 56 & 56 & 57 & 57 & 58 & 58 & 57 & 58 \\
\hline P75 & 54 & 54 & 54 & 55 & 55 & 56 & 55 & 57 \\
\hline P50 & 52 & 51 & 51 & 52 & 53 & 53 & 50 & 54 \\
\hline $\mathrm{P} 25$ & 48 & 47 & 48 & 49 & 49 & 47 & 45 & 50 \\
\hline P10 & 46 & 44 & 45 & 46 & 44 & 44 & 42 & 47 \\
\hline P5 & 43 & 41 & 42 & 43 & 42 & 42 & 39 & 43 \\
\hline
\end{tabular}

Nota. Puntuación máxima: 60 puntos. $\mathrm{N}=979$. 
tados obtenidos.

Considerando que el presente estudio analiza los resultados para el grupo etario 19-30 años, es decir, quienes nacieron entre 1988 y 1999, en este trabajo nos ocupamos de las generaciones Y, o millennials, y Z, o centennials. La generación Y o millennials es la primera integrada por nativos digitales. Se constituye sustancialmente a partir de la aparición de internet y la globalización, expresándose en una conectividad a escala global y cuya velocidad equivale a la inmediatez. Todos son nativos digitales, y la brecha generacional con los grupos anteriores no se circunscribe exclusivamente a cuestiones del orden social, histórico, tecnológico, simbólico específicas de cada grupo, sino que también supone una brecha cognitiva. En ese sentido, las modificaciones en las capacidades cognitivas que postulan dichos autores, signadas por la inmediatez, podrían contribuir al detenimiento del efecto Flynn. Pensemos que estos tests son tomados en el contexto áulico, para los menores de 18 años, y en universidades e institutos terciarios para los mayores. Con esto queremos señalar que quienes realizan la prueba no lo hacen condicionados por el futuro resultado, como podría pasar en una entrevista laboral, por ejemplo. Esto nos hace pensar que quienes están resolviendo la prueba podrían cuestionar la motivación para llevar adelante el esfuerzo que conlleva esta tarea. Tal vez aparezcan dificultades para concentrarse durante varios minutos seguidos en una misma tarea, sumándose a ello las ansiedades por obtener un resultado de manera inmediata. Estas condiciones pueden caracterizar a los sujetos que son evaluados con el Test de Raven, y reflejarse directamente sobre los resultados finales.

Posibles líneas de investigación futura podrían incluir la indagación de si existe un correlato, en otros aspectos de la inteligencia ligados a la motricidad y el lenguaje, de la mencionada diferencia cognitiva intergeneracional observada a nivel de la capacidad eductiva.

\section{Referencias}

Armstrong, E. L., \& Woodley, M. A. (2014). The rule-dependence model explains the commonalities between the Flynn effect and IQ gains via retesting. Learning and Individual Differences, 29, 41-49. doi: 10.1016/j.lindif.2013.10.009

Baker, D. P., Eslinger, P. J., Benavides, M., Peters, E., Dieckmann, N. F., \& Leon, J. (2015). The cognitive impact of the education revolution: A possible cause of the Flynn effect on population IQ. Intelligence, 49, 144-158. doi: 10.1016/j.intell.2015.01.003

Bauman, Z. (2002). Modernidad líquida. Buenos Aires, Argentina: Fondo de Cultura Económica.

Bauman, Z. (2008). Los retos de la educación en la modernidad líquida. Barcelona, España: Gedisa.

Bratsberg, B., \& Rogeberg, O. (2018). Flynn effect and its reversal are both environmentally caused. Proceedings of the National Academy of Sciences of the United States of America, 115(26), 6674-6678. doi: 10.1073/pnas.1718793115

Brouwers, S. A., van de Vijver, F. J., \& van Hemert, D. A. (2009). Variation in Raven's Progressive Matrices scores across time and place. Learning and Individual Differences, 19(3), 330-338. doi: 10.1016/j.lindif.2008.10.006

Di-Rienzo, J. A., Casanoves, F., Balzarini, M. G., Gonzalez, L., Tablada, M., \& Robledo, C. W. (2018). InfoStat (versión 2018) [software de cómputo]. Recuperado de http://www.infostat.com.ar

Dutton, E., Bakhiet, S. F. A., Alhossein, A. H., \& Essa, Y. A. S. (2019). A Flynn effect in Kuwait, 1985-1998. Personality and Individual Differences, 138, 355-357. doi: 10.1016/j.paid.2018.10.029

Dutton, E., Bakhiet, S. F. A., Osman, H. A., Becker, D., Essa, Y. A. S., Blahmar, T. A. M., \& Hakami, S. M. (2018). A Flynn effect in Khartoum, the Sudanese 
capital, 2004-2016. Intelligence, 68, 82-86. doi: 10.1016/j.intell.2018.03.007

Dutton, E., Essa, Y. A. S., Bakhiet, S. F., Ali, H. A. A., Alqafari, S. M., Alfaleh, A. S. H., \& Becker, D. (2018). Brain drain in Syria's ancient capital: No Flynn effect in Damascus, 2004-2013/14. Personality and Individual Differences, 125, 10-13. doi: 10.1016/j. paid.2017.12.025

Fernández-Liporace, M., Ongarato, P., Saavedra, E., \& Casullo, M. M. (2004). El Test de Matrices Progresivas, Escala General: Un análisis psicométrico. Revista Evaluar, 4, 50-69. Recuperado de https://revistas. unc.edu.ar/index.php/revaluar/index

Flynn, J. R. (1984). The mean IQ of Americans: Massive gains 1932-1978. Psychological Bulletin, 95(1), 2951. doi: 10.1037/0033-2909.95.1.29

Flynn, J. R. (2013). The "Flynn effect" and Flynn's paradox. Intelligence, 41(6), 851-857. doi: 10.1016/j. intell.2013.06.014

Flynn, J. R., \& Rossi-Casé, L. (2011). Modern women match men on Raven's Progressive Matrices. Personality and Individual Differences, 50(6), 799-803. doi: 10.1016/j.paid.2010.12.035

Flynn, J. R., \& Rossi-Casé, L. (2012). IQ gains in Argentina between 1964 and 1998. Intelligence, 40(2), 145150. doi: 10.1016/j.intell.2012.01.006

Gignac, G. E. (2015). Raven's is not a pure measure of general intelligence: Implications for $g$ factor theory and the brief measurement of $g$. Intelligence, 52, 7179. doi: 10.1016/j.intell.2015.07.006

Hartshorne, J. K., \& Germine, L. T. (2015). When does cognitive functioning peak? The asynchronous rise and fall of different cognitive abilities across the life span. Psychological Science, 26(4), 433-443. doi: 10.1177/0956797614567339

Hayes, T. R., Petrov, A. A., \& Sederberg, P. B. (2015). Do we really become smarter when our fluid-intelligence test scores improve? Intelligence, 48, 1-14. doi: 10.1016/j.intell.2014.10.005

Instituto Nacional de Estadística y Censos. (2012). Censo Nacional de Población, Hogares y Viviendas 2010.
Censo del Bicentenario. Resultados definitivos. Serie $\mathrm{B} \mathrm{N}^{\circ} 2$. Tomo 1. Buenos Aires, Argentina: Autor. Recuperado de https://www.indec.gob.ar

Microsoft Corporation. (2010). Microsoft Office Excel (Versión 2010) [software]. Redmond, WA: Microsoft.

Navós. O. T. (2014). Nuevas generaciones en universidades privadas: ¿Qué hacer? Algunas propuestas para la gestión desde el punto de vista del marketing. Buenos Aires: Dunken.

Passig, D. (2015). Revisiting the Flynn effect through 3D immersive virtual reality (IVR). Computers \& Education, 88, 327-342. doi: 10.1016/j.compedu.2015.05.008

Piscitelli, A. (2006). Nativos e inmigrantes digitales. ¿Brecha generacional, brecha cognitiva, o las dos juntas y más aún? Revista Mexicana de Investigación Educativa, 11(28), 179-185. Recuperado de https://www. comie.org.mx/revista/v2018/rmie/index.php/nrmie

Prensky, M. (2001). Digital natives, digital immigrants part 1. On the Horizon, 9(5), 1-6. doi: 10.1108/10748120110424816

Raven, J. C. (2005). Test de Matrices Progresivas, Escala General, Carpeta de Evaluación. Buenos Aires, Argentina: Paidós.

Raven, J. C., Raven, J., \& Court, J. H. (2003). Test de Matrices Progresivas: Escala General. Buenos Aires, Argentina: Paidós.” Test de Matrices Progresivas: Escala General. Buenos Aires, Argentina: Paidós.” Test de Matrices Progresivas: Escala General. Buenos Aires, Argentina: Paidós.

Rindermann, H., \& Becker, R. (2018). Flynn-effect and economic growth: Do national increases in intelligence lead to increases in GDP? Intelligence, 69, 8793. doi: 10.1016/j.intell.2018.05.001

Rindermann, H., Becker, D., \& Coyle, T. R. (2017). Survey of expert opinion on intelligence: The Flynn effect and the future of intelligence. Personality and Individual Differences, 106, 242-247. doi: 10.1016/j. paid.2016.10.061

Rossi-Casé, L., Doná, S. M., Biganzoli, B., \& Garzaniti, R. 
(2019). Evaluando a los Millennials. Apreciaciones sobre la inteligencia a partir del Test de Raven. Perspectivas en Psicología, 16(1), 14-25. Recuperado de http://200.0.183.216/revista/index.php/pep/issue/ view/24

Rossi-Casé, L., Doná, S. M., Garzaniti, R., Biganzoli, B., \& Llanos-Barja, C. (2018). La inteligencia a través de las generaciones: Millennials y centennials. Acta de Investigación Psicológica, 8(2), 90-100. Recuperado de http://www.psicologia.unam.mx/acta-de-investigacion-psicologica

Rossi-Casé, L., Neer, R., \& Lopetegui, S. (2001). Test de Matrices Progresivas de Raven: Comparación de baremos. El aumento de los puntajes directos a través del tiempo. Revista Evaluar, 1, 39-51.

Rossi-Casé, L., Neer, R., \& Lopetegui, S. (2002). Test de Matrices Progresivas de Raven: Construcción de baremos y constatación del "efecto Flynn". Orientación y Sociedad, 3, 181-187. Recuperado de http:// www.psico.unlp.edu.ar/articulo/2015/11/16/revista_orientacion_y_sociedad

Rossi-Casé, L., Neer, R., \& Lopetegui, S. (2017). Normas de la ciudad de La Plata, Argentina (2000 y 20032004). En J. C. Raven (Ed.), Test de Matrices Progresivas. Carpeta de Evaluación, Escala General, Normas (pp. 21-25). Buenos Aires, Argentina: Paidós.

Rossi-Casé, L., Neer, R., Lopetegui, S., Doná, S. M., Biganzoli, B., \& Garzaniti, R. (2014). Matrices Progresivas de Raven: Efecto Flynn y actualización de baremos. Revista de Psicología, 23(2), 3-13. doi: 10.5354/0719-0581.2014.36144

Rossi-Casé, L., Neer, R., Lopetegui, S., Doná, S., Biganzoli, B., \& Garzaniti, R. (2016). Test de Raven: Actualización de baremos en adolescentes argentinos y análisis del efecto Flynn. Revista Iberoamericana de Diagnóstico y Evaluación - e Avaliação Psicológica, 42(2), 3-13 . doi: 10.21865/RIDEP42_3

Sartori, G. (1998). Homovidens. La sociedad teledirigida. Buenos Aires, Argentina: Taurus.

Strauss, W., \& Howe, N. (1991). Generations. The History of America's Future, 1584 to 2069. New York, NY: William Morrow.

Sundet, J. M., Barlaug, D. G., \& Torjussen, T. M. (2004). The end of the Flynn Effect? A study of secular trends in mean intelligence test scores of Norwegian conscripts during half a century. Intelligence, 32(4), 349-362. doi: 10.1016/s0160-2896(04)00052-2

Teasdale, T. W., \& Owen, D. R. (2007). Secular declines in cognitive test scores: A reversal of the Flynn effect. Intelligence, 36(2), 121-126. doi: 10.1016/j.inte11.2007.01.007

Wechsler, D. (2008). Wechsler Adult Intelligence Scale (4 ed.). San Antonio, TX: Pearson Assessment. doi: 10.1037/t15169-000 